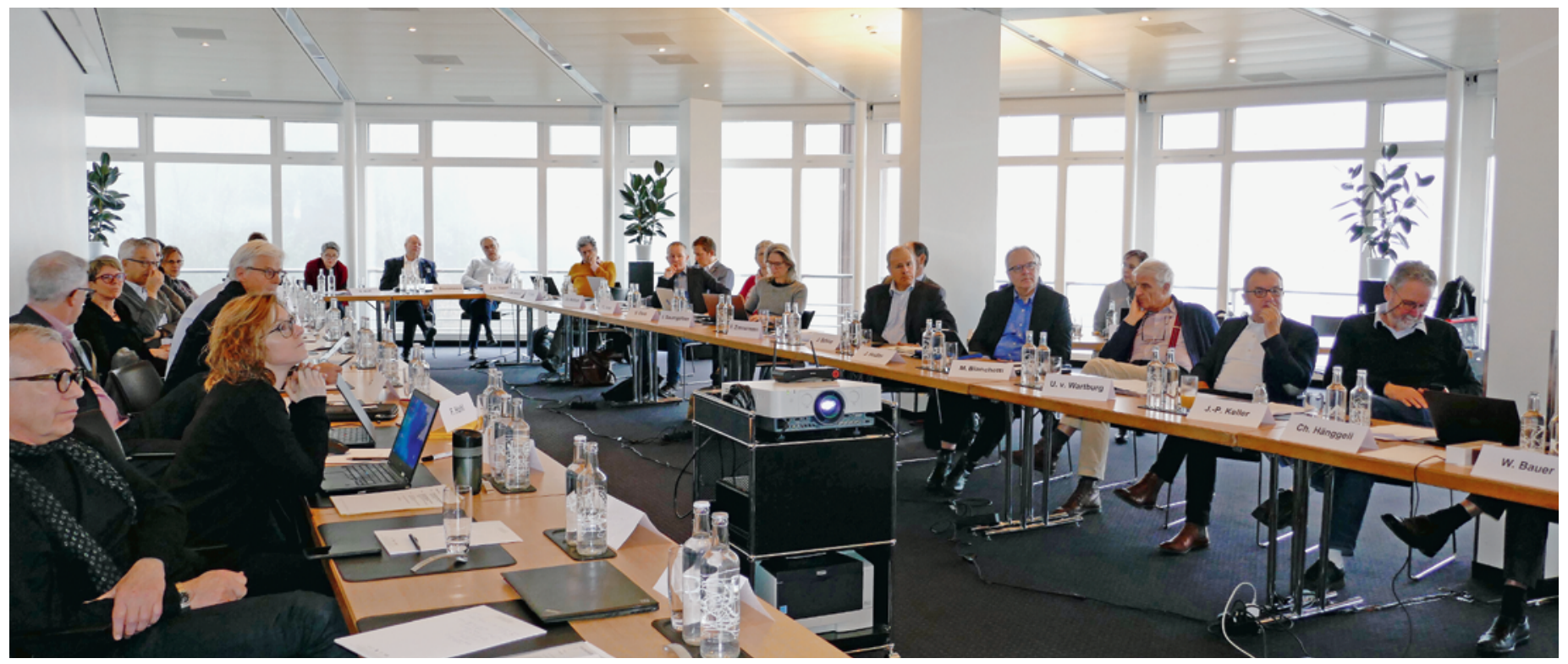

\title{
Die Digitalisierung in die ärztliche Bildung integrieren
}

\section{Adrian Rittera, Fabienne Hohl ${ }^{b}$}

a Freischaffender Journalist, vor Ort am 24.1.2020; b Texterin, Berichterstattung vom 25.1.2020

Die jährliche Journée de réflexion von SIWF und Collège des Doyens ermöglicht den Austausch über Fragen der ärztlichen Fort- und Weiterbildung. An Themen mangelte es auch in diesem Jahr nicht - von der Finanzierung bis zur Künstlichen Intelligenz.

Freiraum zum Nachdenken über Bildung: Mit diesem Ziel trafen sich Ende Januar in Luzern rund 40 Fachleute zur jährlichen Journée de réflexion. Organisiert war der Anlass von Werner Bauer, Präsident des Schweizerischen Instituts für ärztliche Weiter- und Fortbildung (SIWF) und Hans-Uwe Simon, Dekan der

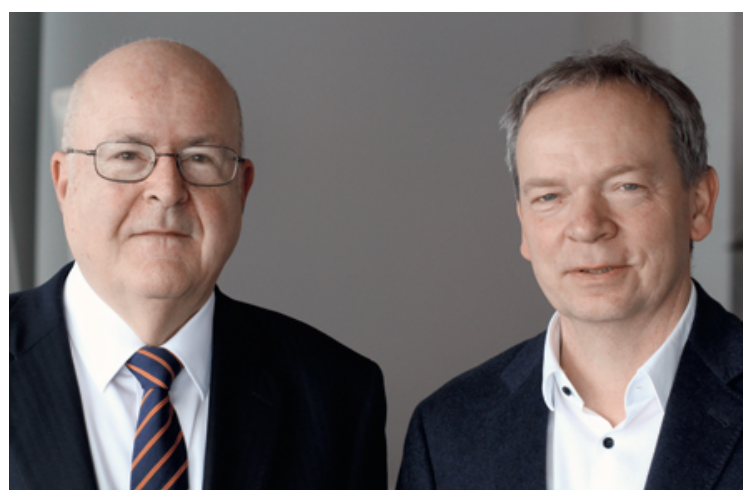

Werner Bauer (links) und Hans-Uwe Simon
Medizinischen Fakultät der Universität Bern und Präsident des Collège des doyens.

Das Collège durfte im vergangenen Jahr neue Mitglieder aufnehmen, da unter anderem die Universitäten Fribourg, St.Gallen und Luzern neu ebenfalls medizinische Master-Studiengänge anbieten. Weiter berichtete Hans-Uwe Simon, dass im Collège derzeit ein Antrag diskutiert werde, der vorsieht, dass die Medizinischen Fakultäten der Schweiz gegenseitig Habilitationen vereinfacht anerkennen.

Die vom SIWF angebotene elektronische Fortbildungsplattform werde weiter ausgebaut, informierte Werner Bauer. In Zukunft werde es möglich sein, über die Plattform unter anderem Credits zu vergeben und Veranstaltungen zu bewerten. Zurzeit bearbeite das SIWF auch ein Mandat des Bundesamtes für Gesundheitswesen und der Gesundheitsdirektorenkonferenz. Ziel sei es, den aktuellen Stand der ärztlichen Fortbildung in der Schweiz zu eruieren und allfällige Vorschläge 
zur Qualitätsverbesserung auszuarbeiten. Der Schlussbericht dazu soll bis im September 2020 vorliegen.

Ein weiteres Thema, welches das SIWF beschäftigt, ist die Ausrichtung des Medizinstudiums auf Entrustable Professional Activities (EPA). Diese gelte es jetzt auch in die Weiterbildung zu integrieren, was eine anspruchsvolle Aufgabe sei, so Bauer. Das SIWF habe eine entsprechende Arbeitsgruppe geschaffen, eine Arbeitstagung für die Fachgesellschaften organisiert und sei dabei, ein Positionspapier und Leitlinien zu erarbeiten.

\section{Viele ausländische Diplome und Facharzttitel}

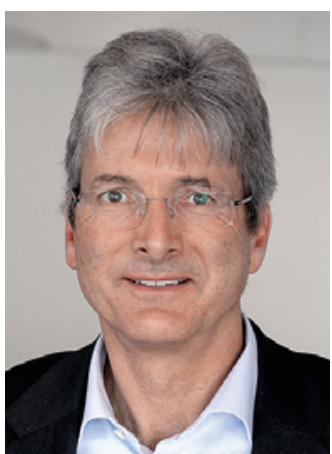

Christoph Hänggeli
Christoph Hänggeli präsentierte als Geschäftsführer des SIWF die aktuellen Zahlen zur medizinischen Bildungslandschaft. Das Institut habe im vergangenen Jahr 1334 Facharzttitel vergeben. Dies entspreche einer leicht tieferen Zahl als im Vorjahr, obwohl eine Steigerung erwartet worden sei. Gleichzeitig nehme nämlich die Zahl der Assistentenstellen kontinuierlich zu. Die Eidgenössische Medizinalberufekommission habe im vergangenen Jahr zusätzlich 2940 ausländische Arztdiplome und 1280 ausländische Facharzttitel anerkannt. Auffallend: Insbesondere in Fachbereichen wie der Plastischen Chirurgie, der Herzchirurgie und der Urologie liege die Zahl der anerkannten ausländischen Facharzttitel deutlich höher als die Zahl der erteilten Titel aufgrund einer Facharztweiterbildung in der Schweiz.

\section{Erschwerende Umstände}

Jürg Hodler, Ärztlicher Direktor des UniversitätsSpitals Zürich, reflektierte in seinem Referat am Beispiel eines akademischen Spitals, wie sich strukturelle Veränderungen auf die ärztliche Weiterbildung auswirken. Die medizinische Aus-, Fort- und Weiterbildung in der Schweiz sei grundsätzlich gut, betonte er. Allerdings ergebe es äussere Umstände wie Regulierungen und Marktveränderungen, welche die Weiterbildung erschwerten.

So bedingten neue gesetzliche Bestimmungen wie das Heilmittelgesetz, dass die entsprechenden Inhalte an die Assistenzärztinnen und Assistenzärzte vermittelt werden. Dieser zeitliche Aufwand gehe von der übrigen Weiterbildungszeit ab. Die politisch vorgegebene

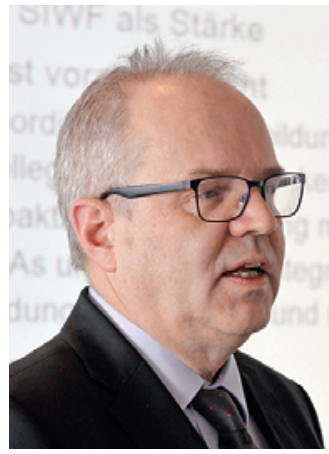

Jürg Hodler
Ausrichtung «ambulant vor stationär» führe zudem dazu, dass auch die Weiterbildung vermehrt im ambulanten Rahmen stattfinde. Dort stehe dafür weniger Zeit zur Verfügung, zumal ambulante Leistungen tiefer entschädigt werden.

Im Bereich der Marktveränderungen erwähnte Hodler den steigenden Anspruch an Perfektion, bisweilen auch juristisch ausgetragen. Dies führe dazu, dass Spitäler vorsichtiger würden und vermehrt Kaderärzte für Behandlungen einsetzten - was nicht im Interesse der Weiterbildung unterer Stufen sei.

\section{Frauenanteil erhöhen}

Ein Fragezeichen setzte Hodler hinter den Lernzielkatalog für das Studium PROFILES: Dieser bringe zwar eine wünschenswerte Anpassung an moderne Lehrmethoden, behandle aber wissenschaftliche Themen zu wenig. Diese Ent-Akademisierung der Medizin sei eine Gefahr. Es brauche mehr Hausärzte, aber auch mehr klinisch forschende Medizinerinnen und Mediziner. Weiter forderte Hodler, die ärztliche Führungsausbildung und die Förderung der Gleichstellung zu verstärken. Das UniversitätsSpital Zürich arbeite daran, den Frauenanteil auf der Stufe "Leitende Ärzte» von heute rund 20 auf 30 Prozent zu erhöhen.

Da die grossen Spitäler einen massgeblichen Teil der ärztlichen Weiterbildung tragen, sollten sie die Weiterbildungsprogramme vermehrt mitprägen können, sagte Hodler. Nötig sei eine standardisierte, innovative und überprüfte Vorbereitung der Assistenzärzte, bevor sie Patientinnen und Patienten behandeln. Dabei könnten Weiterbildungen vermehrt von spezialisiertem Wissen entlastet werden, wie Hodler in seinen Thesen formulierte.

In der anschliessenden Diskussion wurde unter anderem «Protected Research Time» als Möglichkeit erwähnt, der Ent-Akademisierung entgegenzuwirken. «Protected Time» könnte auch ein Modell sein, um Oberärzten zu ermöglichen, für eine gewisse Stundenzahl pro Woche als «Lehroberärzte» mit speziellen Aufgaben in der Weiterbildung tätig zu sein. Weitere Ideen aus dem Plenum betrafen den vermehrten Einsatz von Simulationen in der Weiterbildung. $\mathrm{Zu}$ diskutieren seien zudem Ausgleichszahlungen von Spitälern, die weniger Aufgaben in der Weiterbildung übernehmen. 


\section{Die Gesundheitsversorgung neu denken}

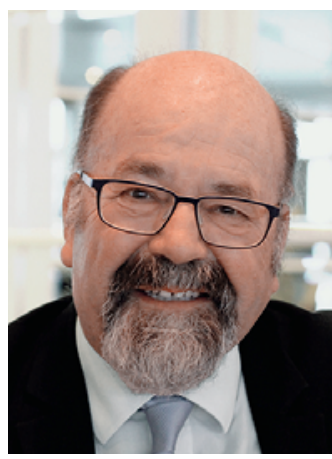

Joachim Eder
Mit den Rahmenbedingungen für die ärztliche Bildung setzte sich als nächster Referent auch Joachim Eder auseinander. Als ehemaliger FDP-Ständerat und Präsident der Kommission für soziale Sicherheit und Gesundheit sowie ehemaliger Gesundheitsdirektor des Kantons Zug stellte er fest: Wachsender Kosten-

druck, steigende Qualitätsanforderungen und Fachkräftemangel bedingen, die Gesundheitsversorgung neu zu denken. Dabei sei auch eine Reduktion der Zahl der Spitäler unumgänglich, wobei heute mit insgesamt 70 Spital-Investitionsprojekten noch das Gegenteil stattfinde.

Die ärztliche Aus-, Fort- und Weiterbildung stehe in der derzeitigen politischen Diskussion zwar nicht im Zentrum, sei aber doch Gegenstand zahlreicher parlamentarischer Vorstösse. So verlange ein Postulat, die Tendenz zu immer mehr ausländischen Ärztinnen und Ärzten umzukehren. Und eine hängige Motion fordert, die Anschubfinanzierung für ärztliche Ausbildungsplätze in der Humanmedizin weiterzuführen. Eder zeigte sich wenig begeistert von der Idee, eine «Anschubfinanzierung» auf Bundesebene zu verlängern - zumal die Aufgabe eigentlich auf die Ebene der Kantone gehöre. Was die Finanzierung der ärztlichen Weiterbildung anbelangt, sieht eine interkantonale Vereinbarung Ausgleichszahlungen zwischen den Kantonen vor. Das Konkordat tritt allerdings erst in Kraft, wenn ihr mindestens 18 Kantone zustimmen. Gemäss Eder wird das Geschäft im Laufe des Jahres 2020 in mehreren kantonalen Parlamenten behandelt. Er gab sich überzeugt, dass die Vereinbarung zustande kommt. Eine weitere Idee könnte gemäss Eder sein, die Weiterbildung in den Leistungsaufträgen mit Spitälern konkret zu verankern oder als gemeinwirtschaftliche Leistungen anerkennen zu lassen.

\section{Digitalisierung als Chance}

Die zweite Hälfte der Journée de réflexion war der Digitalisierung und ihren Auswirkungen auf den Arztberuf gewidmet. Zu Beginn skizzierte Zukunftsforscher Daniel Dettling mit Optimismus, wie sich Technologie und demographischer Wandel produktiv zusammenführen lassen. Die Digitalisierung habe das Potenzial, die Arbeitswelt menschlicher zu machen, indem sie zuverlässig Routinetätigkeiten übernehme und Trans-

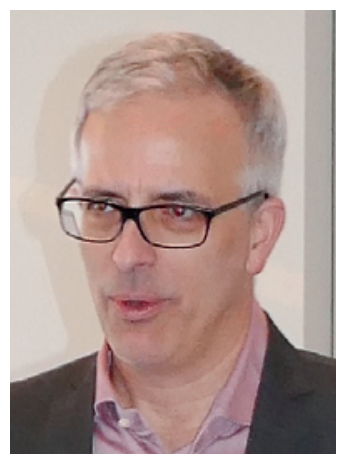

Daniel Dettling parenz schaffe. Sie werde den Arztberuf keineswegs überflüssig machen, betonte Dettling, aber sie werde ihn verändern. Denn die Menschen machen sich die neuen Technologien zunutze, um ihre Gesundheit in eigener Kompetenz zu stärken. Dies zeigt sich etwa im zunehmenden Angebot der GesundheitsFerner hat sich die Telemedizin bereits gut etabliert, macht sie doch schon heute ein gutes Drittel der ärztlichen Konsultationen aus.

Wir werden heute immer älter, gesünder und technikaffiner. Angesichts dieser Tatsache postulierte Dettling, die Gesellschaft solle sich insbesondere auf die Bedürfnisse der lebensfrohen Silver Agers einstellen - statt Notstandsmassnahmen gegen die Überalterung zu ergreifen. Der Zukunftsforscher zeigte sich überzeugt, dass die veränderten Ansprüche moderner Menschen viele neue Angebote hervorrufen werden, etwa in Bereichen wie «Wissensnavigation" und "Gesundheitsassistenz». So könnte die Medizin dem nachgewiesenen Wunsch nach dem vertrauensvollen virtuellen Austausch mit Gesundheitsexperten entsprechen. Aus dem Publikum kam der Einwand, dass die dank Digitalisierung eingesparte Zeit weniger den Patienten zugutekäme als vielmehr einem noch höheren Arbeitstakt. Dagegen müsse man sich entschieden wehren, sagte Dettling und versicherte, dass sich die Ärzteschaft dabei auf die gute Lobby ihrer Patientinnen und Patienten verlassen könne.

\section{Angewandte Virtual Reality}

Dem kulturwissenschaftlichen Input zur Digitalisierung folgte ein ebenso spannender Einblick in die Praxis der virtuellen Realität (VR). Philippe Cattin vom

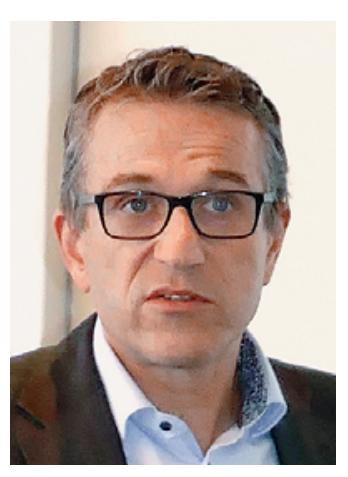

Philippe Cattin Department of Biomedical Engineering der Universität Basel referierte über die dreidimensionale Simulation von Körpern. VR-Darstellung habe grosse Vorteile gegenüber realen Modellen: Sie sei schnell, intuitiv erfassbar, bilde reale Patientendaten ab und sei beliebig vielen Betrachtenden zugänglich - auch 
unabhängig von deren Aufenthaltsort. Gerade Letzteres sei für den fachlichen Austausch unschätzbar.

Aktuell liessen sich Knochen und Gefässe bereits gut darstellen, Gewebe hingegen seien noch eine Herausforderung, führte Cattin aus. Doch schon heute sei die Simulation realer Körper ein verdienstvolles Instrument in der Aus- und Weiterbildung, nützlich zur Planung von Operationen, für die Patientenaufklärung sowie für erste Versuche, bei Operationen schonenden Laser statt Skalpelle einzusetzen. Als nächste Stufe der VR-Simulation beschrieb Cattin das Integrieren von haptischem Feedback via Robotik. Damit könne in naher Zukunft das virtuelle Training beispielsweise in der Chirurgie noch intuitiver und individueller gestaltet werden.

Wie bestechend gut schon eine einfache, mobile Variante des Simulationsprogramms der Basler Universität funktioniert, konnten die Gäste der Journée de réflexion gleich selber testen: Dabei stellt eine 3DBrille einen menschlichen Schädel mit 180 Bildern pro Sekunde dar, was den Betrachtenden eine angenehm ruhige, flimmerfreie Ansicht ermöglicht. Die Simulation lässt sich mittels Steuerung beliebig im Raum wenden und drehen, und noch besser: aus jeder Perspektive virtuell betreten. Eine faszinierende Erfahrung.

\section{KI verstehen lernen}

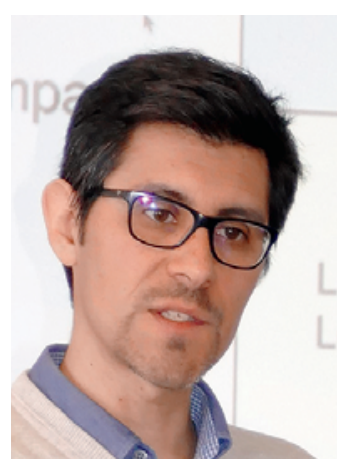

Mauricio Reyes

Künstliche Intelligenz (KI) und die Rolle, welche sie in der ärztlichen Bildung spielt, war das Thema von Mauricio Reyes. Der Computerspezialist vom Inselspital Bern erörterte, weshalb digitale Datenverarbeitung so oft auf Widerstand stösst bei all ihren grundsätzlichen Vorzügen als präzises, effizientes und unermüdli-

ches Arbeitsmittel. Reyes nannte die Mängel von KI beim Namen: beispielsweise schlechte Datenqualität, die entsprechend enttäuschende Resultate zeitige, Kinderkrankheiten bei neuen Entwicklungen oder die fehlende Kompatibilität zwischen verschiedenen Informa- tiksystemen. Gleichzeitig erinnerte Reyes an den Zeitgewinn, den die Technologie zugunsten einer menschlicheren Medizin schaffen könne, und brach eine Lanze für alle Bemühungen, KI zu vermitteln und für die Medizin fruchtbar zu machen. Ärztinnen und Ärzte müssten die neuen Technologien verstehen, hielt er fest. Nur so könnten sie ihnen vertrauen und von ihnen profitieren.

Reyes' Anregung, KI-Themen regelmässig und auch niederschwellig in die ärztliche Bildung zu integrieren, stiess beim Publikum auf offene Ohren. Dabei wurde aber auch auf die oftmals ungenügende IT-Ausrüstung in den Institutionen hingewiesen. Zudem wurden Befürchtungen laut, dass neue Inhalte die Ausbildung über Gebühr verlängern könnten. Worauf Werner Bauer die EPA ins Spiel brachte. Sie seien eine vielversprechende Methodik, in deren Rahmen sich Studierende und Weiterzubildende auch fachrelevantes KI-Wissen effizient aneignen könnten.

\section{Ausblick}

Im Übrigen behaupteten sich die EPA auch dieses Jahr auf der traditionellen To-do-Liste, die Werner Bauer zu Ende der Journée de réflexion präsentierte. Den EPA gehöre die Zukunft unter anderem auch deswegen, weil sie sich an der tatsächlichen, individuellen Lerndauer für einzelne ärztliche Tätigkeiten orientierten, hielt Werner Bauer fest. Des Weiteren will das SIWF stärker mit den Weiterbildungsstätten zusammenarbeiten, wenn es darum geht, die Weiterbildungsprogramme gezielt zu aktualisieren. Stichworte hierzu waren Wahlmodule, koordinierte KI-Angebote für mehrere Fachgebiete oder die Anregung, für jede Kategorie von Weiterbildungsstätten in den Weiterbildungsprogrammen eine gewisse Anzahl von Lehrarztstellen im Kader vorzuschreiben. Diese Idee wurde ausdrücklich begrüsst, denn eine enge Begleitung gerade der frisch gebackenen Masters of Medicine im ersten Halbjahr der Weiterbildung sei für alle Beteiligten eine Notwendigkeit und ein Gewinn.

Einigkeit herrschte darüber, dass für eine gewisse Steuerung der Wahl eines Fachgebiets vor allem Anreize nötig seien - auch finanzielle. Die Journée de réflexion schloss mit der Bekräftigung, dass die ärztliche Bildung KI systematisch und fachgerecht berücksichtigen müsse. Denn die Inputs und Diskussionen der Tagung hatten klar gezeigt: KI-Wissen ist eine unabdingbare Voraussetzung dafür, dass künftige Ärztinnen und Ärzte den digitalen Fortschritt tatsächlich in den Dienst einer möglichst menschlichen Medizin stellen können.

Bildnachweise

Adrian Ritter und Fabienne Hohl 